\title{
99Analysis of Degradation Mechanisms in Donor-Acceptor Copolymer Based Organic Photovoltaic Devices Using Impedance Spectroscopy
}

\author{
Shashi B. Srivastava ${ }^{1}$, Prashant Sonar ${ }^{2}$ and Samarendra P. Singh ${ }^{1 *}$ \\ ${ }^{1}$ Department of Physics, Shiv Nadar University, Gautam Buddha Nagar, \\ Uttar Pradesh, India-201314 \\ ${ }^{2}$ School of Chemistry, Physics and Mechanical Engineering, \\ Queensland University of Technology, Brisbane, Australia \\ (*samarendra.singh@snu.edu.in)
}

\begin{abstract}
The stability of organic photovoltaic (OPV) devices in ambient conditions has been a serious issue which needs to be addressed and resolved timely. In order to probe the degradation mechanism in a donor-acceptor block copolymer PDPP-TNT:PC71BM bulk heterojunction based OPV devices, we have studied current-voltage $(\mathrm{J}-\mathrm{V})$ behaviour and impedance spectroscopy (IS) of fresh and aged devices. The current-voltage characteristic of optimized fresh devices exhibit a short circuit current $\left(J_{s c}\right)$ of $8.9 \mathrm{~mA} / \mathrm{cm}^{2}$, open circuit voltage $\left(V_{o c}\right)$ of $0.79 \mathrm{~V}$, fill factor $(F F)$ of $54.6 \%$, and power conversion efficiency $(P C E)$ of $3.8 \%$. For aged devices, $J_{s c}, V_{o c}, F F$, and $P C E$ was reduced to $42 \%, 10 \%, 56 \%$ and $76 \%$ of its initial value, respectively. The impedance spectra measured under illumination for these devices was successfully fitted using a CPE based circuit model. For aged devices, the low frequency response in impedance spectra suggests an accumulation of the photo-generated charge carriers at the interfaces which impedes the charge extraction at the electrodes and leads to a significant lowering in fill factor. Such degradation in device performance is attributed to the incorporation of oxygen and water molecules in devices. An increase in the recombination resistance associated with the bulk heterojunction active layer indicates a deterioration of free charge carrier generation and conduction in devices. Such observations from impedance measurement appear as an electrical signature of the probable physical mechanisms responsible for degradation of OPV devices.
\end{abstract}

Index Terms - Capacitance-voltage $(\mathrm{C}-\mathrm{V})$ characteristics, charge carrier lifetime, charge transport, impedance spectroscopy, degradation mechanism, lifetime, organic photovoltaic device. 


\section{INTRODUCTION}

During the past decade, bulk heterojunction based organic photovoltaic (OPV) devices have emerged as a potential renewable energy source. OPV devices offer a wide range of advantages like flexibility, lightweight, low cost and high throughput processing [1]. Optimization of device architectures, improvement in device fabrication techniques and continuous development of high performing materials have steered improvement in the power conversion efficiency (PCE) up to $>10 \%$ [2, 3]. In order to establish OPVs competitive with conventional silicon photovoltaic technology, the efficiency and life-time of these devices need to be significantly improved [4, 5].

In OPV devices few degradation mechanisms are known. For example: Diffusion of oxygen and water molecules into the devices, degradation of interfaces, the active materials, inter-layers and electrode materials, electrochemical reactions with the organic materials during device operation and morphological changes [4-7]. However, some of these mechanisms are interrelated and occurs simultaneously which makes it difficult to identify exact degradation mechanisms. Further, it is more difficult to quantify effect of individual degradation mechanism towards the overall degradation of OPV devices. Decay in currentvoltage $(I-V)$ characteristics is typically due to changes in dynamics and recombination kinetics of charge carriers lead by various degradation mechanisms. In order to identify the degradation mechanism and their impact on electrical parameters of devices we need a group of complimentary characterization tools to probe charge carrier transport and their recombination kinetics in the bulk active layer, and at various interfaces in OPV devices.

Impedance spectroscopy (IS) is an effective tool to study transport and recombination mechanisms in the bulk, interfaces and electrodes in OPV devices under working conditions [8]. This technique has been applied to outline the possible degradation mechanisms by comparing the impedance characteristics in fresh and aged devices. Using impedance analysis for ageing OPV devices the formation of an electron barrier at the electrode barrier and role of defects are identified as important degradation mechanisms [9, 10].

In this paper we report an investigation of the degradation mechanism in poly\{3,6dithiophene-2-yl-2,5-di(2-octyldodecyl)-pyrrolo [3,4-c] pyrrole-1,4-dione-alt-naphthalene (PDPP-TNT) and PC71BM based bulk heterojunction inverted OPV devices using impedance spectroscopy. PDPP-TNT is a conjugated structure having naphthalene and diketopyrrolopyrrole (DPP) as donor and acceptor moieties in the polymer backbone, respectively. PDPP-TNT is an interesting hole transporting polymer which has shown 
reasonably good performance in organic field-effect transistors (OFETs) and OPV devices [11]. In this paper, we report a comparative study of internal charge dynamics and recombination kinetics in fresh and aged PDPP-TNT:PC71BM bulk heterojunction based inverted OPV cells. These devices were fabricated and characterized under room ambient. The electrical characteristics and performance of these devices were measured over three weeks under the ambient condition without encapsulation. We have performed currentvoltage $(I-V)$, capacitance-voltage $(C-V)$ measurements and impedance spectroscopy (IS) to study charge carrier transport, their accumulation, and recombination kinetics in PDPPTNT:PC71BM bulk heterojunction based fresh and aged devices.

\section{EXPERIMENTAL DETAILS}

Inverted OPV devices were fabricated on patterned indium tin oxide (ITO) substrates with a configuration of ITO/ZnO/PDPP-TNT:PC71BM/MoO $/$ Ag. The schematic of inverted OPV devices with the molecular structure of PDPP-TNT and PC71BM is shown in Fig. 1. ITO coated glass substrates were cleaned using soap solution (2\% micro-90) in de-ionized (DI) water at $50{ }^{0} \mathrm{C}$ for 20 minutes. The substrates were rinsed with DI water, and sonicated in acetone and isopropanol, respectively. Further, these substrates were dried with dry nitrogen $\left(\mathrm{N}_{2}\right)$ and treated with UV-ozone to induce the hydrophilicity. A sol-gel of $\mathrm{ZnO}(0.45$ M) was prepared using zinc acetate in 2-methoxyethanol and ethanolamine. The prepared solution was spin coated at $2000 \mathrm{rpm}$ for 60 seconds to deposit ZnO thin film ( $40 \mathrm{~nm}$ ) on the substrate. A drying process was performed on a hot plate at $250{ }^{\circ} \mathrm{C}$ for 15 minutes in room ambient [12]. The PDPP-TNT was synthesized as described earlier, and PC71BM was used as it was received from Lumtec, Taiwan. The PDPP-TNT:PC71BM blend layer was deposited by spin-coating a solution (15 mg/ml) of PDPP-TNT (33 wt\%) and PC71BM (67 $\mathrm{wt} \%$ ) in a mixture of chloroform and o-dichlorobenzene (4:1 by volume) at $5000 \mathrm{rpm}$ for 60 seconds on top of ZnO layer. The PDPP-TNT:PC71BM layer was annealed on a hot plate at $60{ }^{0} \mathrm{C}$ for $10 \mathrm{~min}$ to remove the excess solvent $[11,13]$. The thickness of the active layer used in these devices is $150 \mathrm{~nm}$. Molybdenum oxide $\left(\mathrm{MoO}_{\mathrm{x}}\right)$ of $10 \mathrm{~nm}$ thickness, an electron blocking layer, was deposited on top of the active layer by thermal evaporation. The top silver cathode $(100 \mathrm{~nm})$ was subsequently deposited by thermal evaporation through a shadow mask under a pressure of $\sim 2 \times 10^{-6}$ mbar to complete the device, resulting a device area of approximately $0.09 \mathrm{~cm}^{2}$.

Current density-voltage ( $J-V)$ characteristics of OPV devices were measured under an AM1.5G illumination source $\left(1000 \mathrm{Wm}^{-2}\right)$ using a Photo Emission Solar Simulator (Model 
\#SS50AAA). The light intensity was adjusted with a NREL calibrated Si solar cell. The Keithley 4200 SCS parameter analyzer was used for the measurement of $J-V$ characteristics of OPV devices. The impedance analyzer (Autolab PGSTAT-302N) was used for $C-V$ measurement and impedance spectroscopy. Impedance spectra were recorded by applying a small voltage perturbation $(10 \mathrm{mV})$ at frequencies ranging from $1 \mathrm{MHz}$ to $1 \mathrm{~Hz}$.

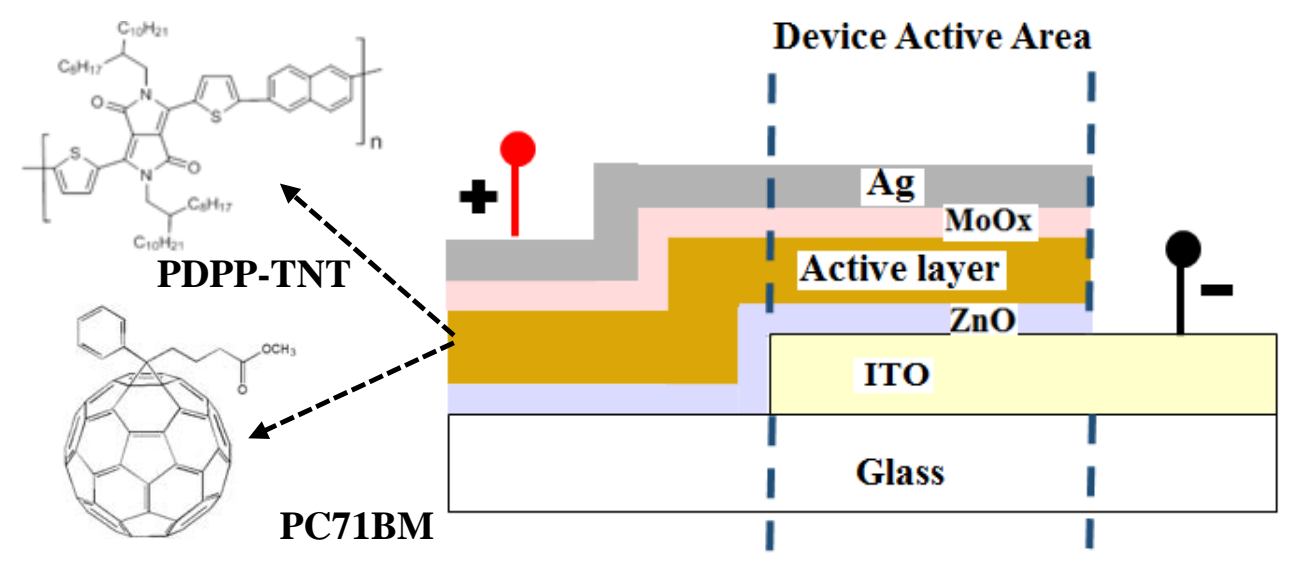

Fig. 1. The structural design of inverted PDPP-TNT:PC71BM bulk heterojunction solar cells with molecular structures of the active layer components.

\section{RESULTS AND DISCUSSIONS}

The $J-V$ characteristics of fresh and aged OPV device, shown in Fig. 2(a) and 2(b) respectively, were measured under dark and light in room conditions. The observed electrical parameters of optimized fresh devices were: a short circuit current $\left(J_{s c}\right)$ of $8.9 \mathrm{~mA} / \mathrm{cm}^{2}$, open circuit voltage $\left(V_{o c}\right)$ of $0.79 \mathrm{~V}$, fill factor $(F F)$ of $54.6 \%$ and power conversion efficiency $(P C E)$ of $3.8 \%$. The same for three weeks aged devices were: a short circuit current $\left(J_{s c}\right)$ of $5.1 \mathrm{~mA} / \mathrm{cm}^{2}$, open circuit voltage $\left(V_{o c}\right)$ of $0.71 \mathrm{~V}$, fill factor $(F F)$ of $24.2 \%$ and power conversion efficiency $(P C E)$ of $0.9 \%$.
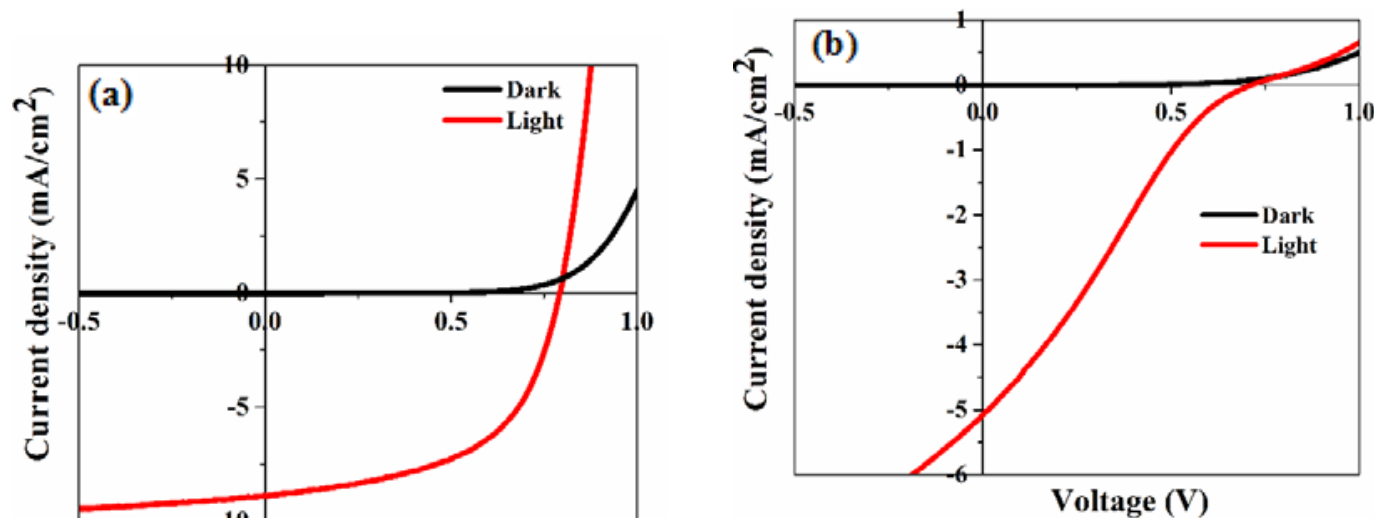
Fig. 2. J-V characteristics of inverted PDPP-TNT:PC71BM bulk-heterojunction solar cells under dark and light measured in room ambient, (a) fresh device, (b) three weeks aged device.

The device parameters were remains constant over two weeks. A significant drop in the device performance parameters was noticed during the third week. For aged devices, $J_{s c}, V_{o c}$, $F F$, and $P C E$ was reduced to $42 \%, 10 \%, 56 \%$ and $76 \%$ of its initial value, respectively. A significant lowering in $J_{s c}$ indicates a decrease in excitons dissociation at PDPP-TNT/PC71BM interface and collection of charge carriers at the electrode [14]. As shown in Fig. 2(b) the aged device could not retain the shape of $J-V$ curve in spite of a small lowering in $V_{o c}$. The $J$ $V$ characteristic for aged device turns to S-shape as shown in Fig. 2(b). In bulk heterojunction devices, such S-shaped $J-V$ curve is reported and attributed to the formation of partially blocking contacts caused by interfacial effects [15]. From the dark $J$ - $V$ characteristic, we noticed that there is no significant change in reverse saturation current. It does indicate that the electrical contacts remain injecting amid degradation of the devices.

With this result, one cannot be sure about the main cause of degradation of the devices with time. In order to get more insight about changes in these devices, we have performed impedance spectroscopy along with $C$ - $V$ analysis for both fresh as well as aged devices.

The $C-V$ characteristic of fresh and aged devices was measured in dark, and under light (1 Sun) as shown in Fig. 3. In fresh devices, a significant increase in the capacitance is noted as compared to the same measured under the dark. This increase in capacitance indicates the modulation of space-charge density and charge accumulation in the device under illumination [16]. The Mott-Schottky (MS) analysis of $C$ - $V$ data shows that the spacecharge density $N_{A} \sim 5.4 \times 10^{16} \# / \mathrm{cm}^{3}$ (under light) as compared to $N_{A} \sim 1.1 \times 10^{17} \# / \mathrm{cm}^{3}$ (under dark). The built-in voltage $\left(V_{b i}\right)$ determined from the intercept of linear fitting results in 0.73 Volts under both dark and light conditions. 

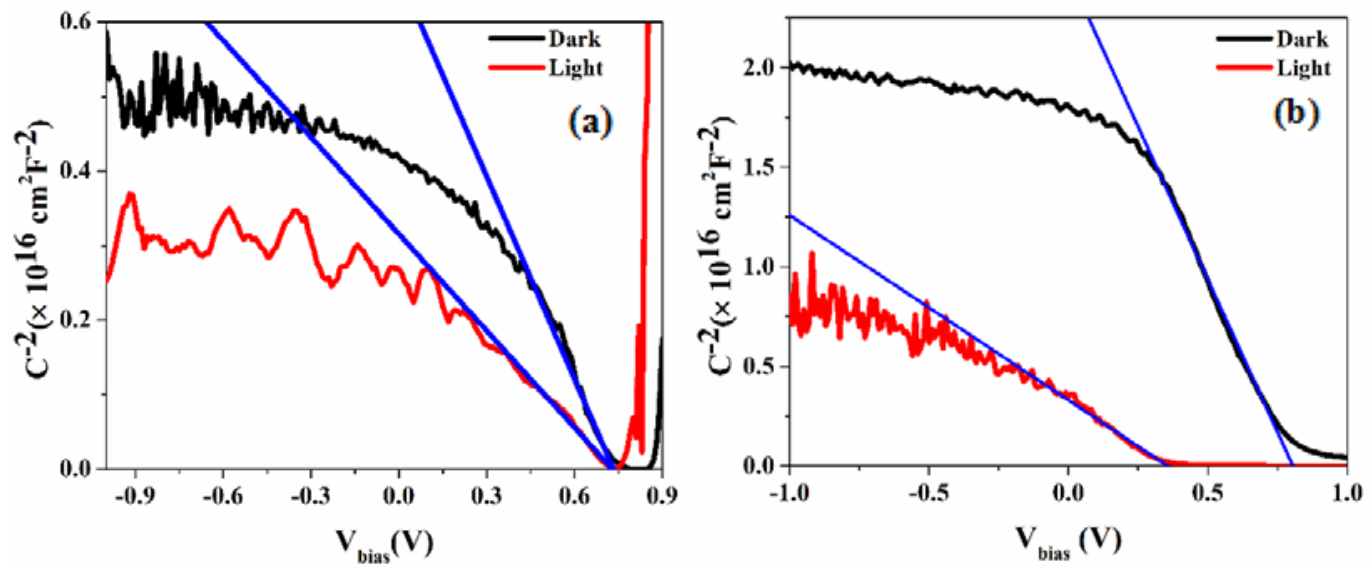

Fig. 3. Comparison of Capacitance-Voltage $(C-V)$ characteristics of (a) fresh and (b) aged devices under dark and light condition.

The space-charge width $(W)$ depends on the space-charge density and the built-in voltage, and can be estimated using following equation,

$$
W=\sqrt{\frac{2 \varepsilon\left(V_{b i}-V\right)}{q N_{A}}}
$$

For fresh devices, the estimated space-charge width under dark and light are $54 \mathrm{~nm}$ and 13 $\mathrm{nm}$, respectively. The higher space-charge density under light implies lower depletion width in devices. This reduction in $W$ changes the energy bands diagram in devices under the light, and affects the built-in electric field, expressed as $E_{b i} \approx\left(V_{b i}-V\right) / W$, present in the space-charge region [17]. Such modulation in built-in field controls the charge-collection in devices. A similar analysis in the case of aged devices results $V_{b i L}=0.34$ Volts and $V_{b i D}=0.77$ Volts. The estimated space-charge width under dark and light are $113 \mathrm{~nm}$ and $70 \mathrm{~nm}$, respectively. Correspondingly, the space-charge density under dark and light are $1.6 \times 10^{16} \mathrm{\#} / \mathrm{cm}^{3}$, and $5.4 \times 10^{16} \mathrm{\#} / \mathrm{cm}^{3}$, respectively.

The space-charge width determined by a bias voltage (0-1 volts) ranges below the thickness of the active layer $(\sim 156 \mathrm{~nm})$ used in these devices. Thick bulk-heterojunction layer in devices ensure reliability of MS analysis on the measured $C$ - $V$ data. The estimated defect level $\left(N_{A} \sim 10^{16}-10^{17} \# / \mathrm{cm}^{3}\right)$ from our $C$ - $V$ data matches well with the same reported by Deledalle et. al. for the donor polymer DPP-TT-T which is based on the same 
diketopyrrolopyrrole (DPP) based functional group, and donor (D)-acceptor (A) coupling scheme to synthesize organic polymers [18].

OPV device under light shows higher capacitance as compared to the same measured in dark. The observed results can be analysed based on depletion region formation at the $\mathrm{BHJ} / \mathrm{ZnO}$ interface [19]. The characteristic capacitance found more in the device under light than the dark, which can be explained as the more charge generation in the bulk than the charge transport to the electrodes and hence accumulation of charges are higher when a device is under operation [20].

In aged devices at certain bias under the light, minority charge carrier concentration increases near the electrode (i.e., electrons at $\mathrm{ZnO}$ ) as compared to the dark conditions which result in more charges at surface states. The extra charge accumulation at surface states enhances the voltage drop in the dipole layer and consequently, lowering in built-in voltage is noticed. Such changes at $\mathrm{BHJ} / \mathrm{ZnO}$ interface also explains S-shape $J-V$ characteristic observed for aged devices [21]. As compared to fresh devices, lower built-in voltage and higher space-charge width noticed in aged devices indicate a redistribution of the built-in electric field in active layer leading to a reduction in $J_{s c}$.

Fig. 4(a) shows the Nyquist plot for fresh device measured under light over the frequency range $1 \mathrm{~Hz}$ to $1 \mathrm{MHz}$, at bias voltage 0.5 to $0.9 \mathrm{~V}$. The impedance spectra comprise of one dominant semicircle and a curved feature observed in low and highfrequency regions, respectively. The high-frequency impedance region is zoomed and shown in Fig. 4(b). The impedance characteristic observed in both frequency regions showed a strong dependence on the bias voltage. In the low-frequency region, with an increase in bias voltage up to $V_{o c}$ the radius of semi-circle decreases rapidly and the depressed semi-circular features are observed. The measured IS data is fitted with the earlier equivalent circuit model as shown in Fig. 4(c). 


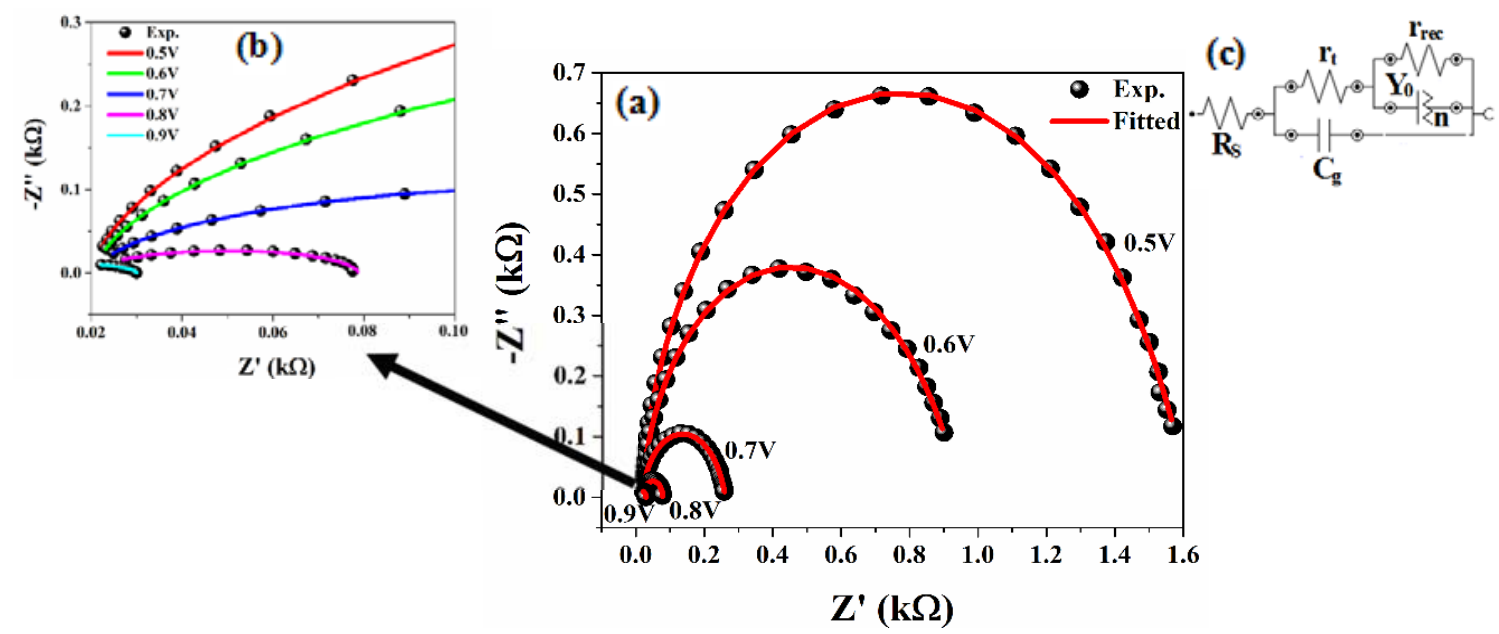

Fig. 4. (a) Impedance spectra of the optimized device measured under light at various bias voltages. Dotted points are experimental data and solid lines are fittings. (b) High-frequency region is shown. (c) The equivalent circuit for the fitting of impedance spectra.

Fig. 5 shown the impedance Cole-Cole diagram for aged device measured under light over the frequency range $1 \mathrm{~Hz}$ to $1 \mathrm{MHz}$, at a bias voltage. The impedance spectra for aged device comprise of one dominant semicircle at low frequency and a second, small and flat semi-circular feature at high frequency, both showing a strong bias voltage dependence. The high-frequency region of the impedance spectra is shown in Fig. 5(b) with decreasing impedance value as bias voltage increases. The impedance behaviour in the low-frequency region at different bias voltage is different from the same for the fresh device. The aged device has increasing impedance with the increase in bias voltage up to $V_{o c}$ and decreasing impedance with increasing bias voltage above $V_{o c}$ as shown in Fig. 5(c) and (d), respectively. Below $\mathrm{V}_{\text {oc}}$, the charge accumulation in the device leads to increase in the semicircle radius with increasing bias voltage.

We have modelled IS experimental data using an equivalent circuit shown in Fig. 4(c), which consist of CPE element (attributed to charge accumulation) in parallel with recombination resistance [22]. The BHJ OPV devices, comprising PDPP-TNT:PC71BM blend layer sandwiched between ITO/ZnO and $\mathrm{MoO}_{\mathrm{x}} / \mathrm{Ag}$ electrodes, is modelled as a parallel combination of a resistor $(R)$ - capacitor $(C)$. Here the capacitor represents a bulk capacitor $\left(C_{g}\right)$ associated with the depletion region inside the active layer and it is equivalent to the geometrical capacitance of the device. The resistor $(R)$ has a combination of bulk resistance $\left(r_{t}\right)$ and recombination resistance $\left(r_{r e c}\right)$ representing resistive characteristics of the active 
layer, and the recombination of photo-generated electrons and holes, respectively. The photogenerated holes and electrons are associated with the acceptor and donor molecules, and this feature can be assigned to a chemical capacitor $\left(C_{\mu}\right)$. In this model, the constant phase element $\left(C_{C P E}\right)$ is used instead of a chemical capacitance, and it covers the aspects of inhomogeneous
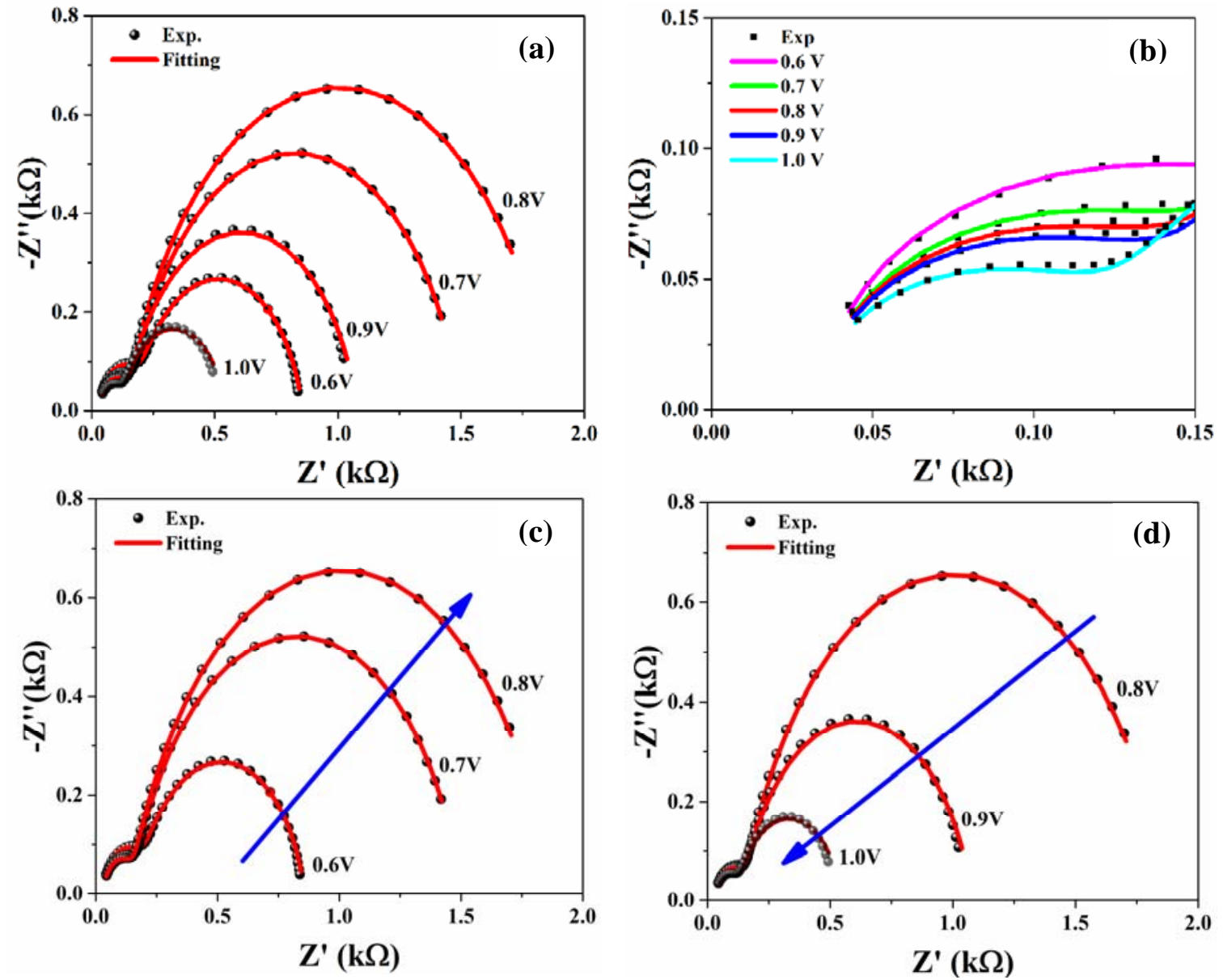

Fig. 5. (a) Impedance spectra of the aged device measured under light at various bias voltages. Dotted points are experimental data and solid lines are fittings. (b) The highfrequency region is shown. (c) Impedance spectra measured under light with bias voltages below $V_{o c}(\mathrm{~d})$ Impedance spectra under light with bias voltages above $V_{o c}$.

nature of interfaces and morphology present in the blended active layer in OPV devices [23]. The electrical resistance present at metal electrodes and electrode/contact layer/active layer interfaces is assigned to a series resistance $R_{s}$. As shown in Fig. 5 the $R-C$ circuit has $r_{t}$ connected in series with $r_{r e c} \| C_{C P E}$ and $C_{g}$ is connected in $\|$ to this combination. Finally, $R_{s}$ is 
connected in series with the $R-C$ circuit. The $r_{r e c} \| C_{C P E}$ combination includes all internal charge dynamical processes and contributes to the response of the device in the lowfrequency region while the $r_{t} \| C_{g}$ combination contributes to the same in the high-frequency region [16, 24].

The random characteristics of a blended active layer in OPV devices can be attributed to the distribution of relaxation times, and is related to impedance as $Z=Y_{0}^{-1}(\mathrm{j} \omega)^{-n}$, where $Y_{0}$ is the coefficient of the CPE and $n$ represents an "ideality" factor characteristic of the distribution of relaxation times. $n$ varies from 0 to 1.0, with 0 corresponding to pure resistive and 1.0 corresponding to pure capacitive behaviour [23].

The recombination resistance $\left(r_{r e c}\right)$ and constant phase element $\left(C_{C P E}\right)$ take into account the recombination of photo-generated holes and electrons, and photo-generated charge carriers (holes on donors and electrons on acceptors) in the inhomogeneous morphology of D-A the blend active layer, respectively. The average charge carrier lifetime $\left(\tau_{n}\right)$ can be estimated using following equations [23]:

$$
C_{\mu}=\frac{\left(Y_{0} r_{r e c}\right)^{\frac{1}{n}}}{r_{r e c}} \quad \text { and } \quad \tau_{n}=r_{r e c} C_{\mu}
$$

The impedance spectra for both fresh and aged devices were fitted using the same model. The ideality factor used in the fitting of the impedance data ranges around 0.54-0.95 which reaffirms capacitive nature of CPE. Due to this reason we represent $C_{C P E}$ by the chemical capacitance $\left(C_{\mu}\right)$, alternatively. The fitted recombination resistance $\left(r_{r e c}\right)$, calculated chemical capacitance $\left(C_{\mu}\right)$ and the electron lifetime using equation (1) are plotted in Fig. 6.

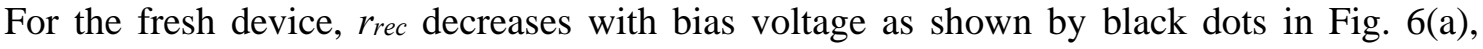
indicating more charge carrier recombination as expected. However, $r_{r e c}$ for the aged devices increases up to $V_{o c}$ and shows decreasing trend above $V_{o c}$ as shown by red dots in Fig. 6(a). An increase of the $r_{r e c}$ (the intercept of the semicircle on the $\mathrm{X}$-axis at low-frequency region) with bias voltage up to $V_{o c}$ can be attributed to a dominant charge accumulation in the device. This suggests that the charge accumulation increases at the interface leading to an increase in the device resistance which explains a significant reduction in fill factor. Fig. 6(b) shows the variation of $C_{\mu}$ with the bias voltage for fresh (black dots) and aged (red dots) device. For the fresh device, $C_{\mu}$ appears almost constant for 0.5 to $0.7 \mathrm{~V}$ and increased for 0.8 and $0.9 \mathrm{~V}$ due to charge injection. However, $C_{\mu}$ for aged devices shows an increase with the bias voltage 
which also confirms accumulation of the photo-generated charge carriers. Fig. 6(c) shows the variation of electron lifetime $\left(\tau_{n}\right)$, a product of $r_{r e c}$ and $C_{\mu}$, with the bias voltage for fresh (black dots) and aged devices (red dots).
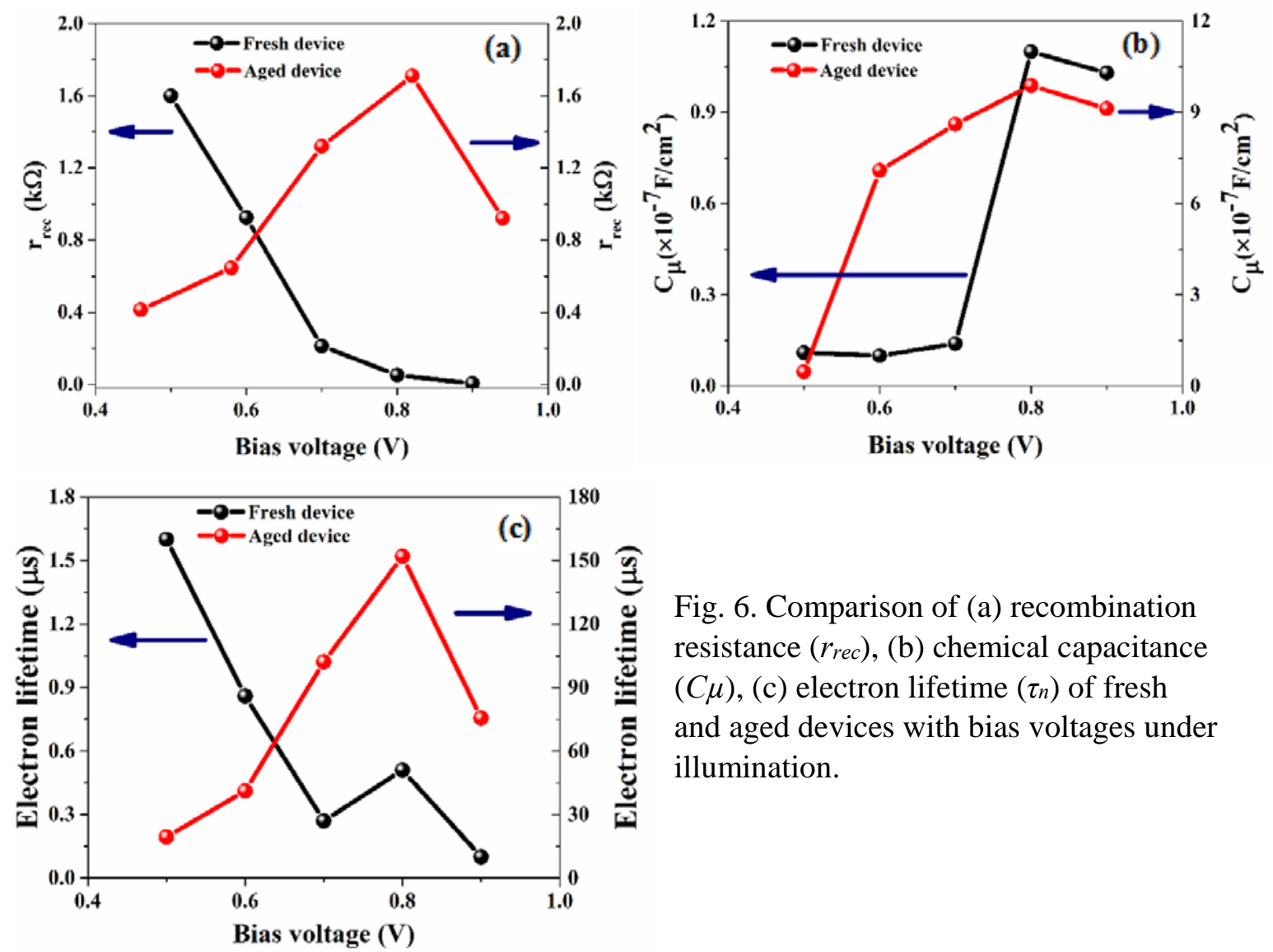

Fig. 6. Comparison of (a) recombination resistance ( $r_{r e c}$ ), (b) chemical capacitance $(C \mu)$, (c) electron lifetime $\left(\tau_{n}\right)$ of fresh and aged devices with bias voltages under illumination.

\section{CONCLUSION}

We have studied current-voltage, capacitance-voltage and impedance spectra of PDPP-TNT and PC71BM based bulk heterojunction inverted OPV devices processed in room ambient. We have characterized these non-encapsulated devices in room ambient for three weeks in order to study degradation. A significant drop in the device performance parameters was noticed during the third week. For aged device $J_{s c}, V_{o c}, F F$, and $P C E$ were reduced to 42 $\%, 10 \%, 56 \%$ and $76 \%$ of its initial value, respectively. The $J-V$ characteristics of the aged device turn to S-shape indicating the formation of partially blocking contacts caused by interfacial effects. The $M-S$ analysis of $C$ - $V$ measurement under illumination suggests lowering in built-in voltage due to the extra charge accumulation at surface states which 
enhances the voltage drop in the dipole layer at $\mathrm{BHJ} / \mathrm{ZnO}$ interface. Such changes at $\mathrm{BHJ} / \mathrm{ZnO}$ interface also explains S-shape $J-V$ characteristic observed for aged devices. The impedance spectra measured under illumination for fresh and aged devices have been successfully fitted using a CPE-based circuit model. Under illumination, the low-frequency response of aged devices in impedance spectra suggests an accumulation of the photogenerated charge carriers at the interfaces which impedes the charge extraction at the electrodes and leads to a significant lowering in fill factor. Such degradation in device performance is attributed to the incorporation of oxygen and water molecules in devices [25]. An increase in the recombination resistance associated with the bulk heterojunction active layer indicates a deterioration of free charge carrier generation and conduction in devices. Such observations from impedance measurement appear as an electrical signature of the probable physical mechanisms responsible for degradation of OPV devices.

\section{References}

[1] N. Grossiord, J. M. Kroon, R. Andriessen, and P. W. M. Blom, "Degradation mechanisms in organic photovoltaic devices," Org. Electron., vol. 13, pp. 432-456, 2012.

[2] M. A. Green, K. Emery, Y. Hishikawa, W. Warta, and E. D. Dunlop, "Solar cell efficiency tables (version 39)," Prog. Photovoltaics Res. Appl., vol. 20, pp. 12-20, 2012.

[3] M. C. Scharber and N. S. Sariciftci, "Efficiency of bulk-heterojunction organic solar cells," Prog Polym Sci, vol. 38, pp. 1929-1940, Dec. 2013.

[4] K. Norrman, N. B. Larsen, and F. C. Krebs, "Lifetimes of organic photovoltaics: Combining chemical and physical characterisation techniques to study degradation mechanisms," Sol. Energ. Mat. Sol. Cells, vol. 90, pp. 2793-2814, Jun. 2006.

[5] M. Jørgensen, K. Norrman, and F. C. Krebs, "Stability/degradation of polymer solar cells," Sol. Energ. Mat. Sol. Cells, vol. 92, pp. 686-714, 2008.

[6] S. Karuthedath, T. Sauermann, H.-J. Egelhaaf, R. Wannemacher, C. J. Brabec, and L. Lüer, "The effect of oxygen induced degradation on charge carrier dynamics in 
P3HT:PCBM and Si-PCPDTBT:PCBM thin films and solar cells," J. Mater. Chem. A, vol. 3, pp. 3399-3408, 2015.

[7] J. Alstrup, K. Norrman, M. Jørgensen, and F. C. Krebs, "Lifetimes of organic photovoltaics: Design and synthesis of single oligomer molecules in order to study chemical degradation mechanisms," Sol. Energ. Mat. Sol. Cells, vol. 90, pp. 27772792, Jun. 2006.

[8] T. K. Mullenbach, Y. Zou, J. Holst, and R. J. Holmes, "Interpreting impedance spectra of organic photovoltaic cells-Extracting charge transit and recombination rates," J. Appl. Phys., vol. 116, p. 124513, 2014.

[9] B. Arredondo, M. B. Martín-López, B. Romero, R. Vergaz, P. Romero-Gomez, and J. Martorell, "Monitoring degradation mechanisms in PTB7:PC71BM photovoltaic cells by means of impedance spectroscopy," Sol. Energ. Mat. Sol. Cells, vol. 144, pp. 422428, 2016.

[10] V. I. Madogni, B. Kounouhéwa, A. Akpo, M. Agbomahéna, S. A. Hounkpatin, and C. N. Awanou, "Comparison of degradation mechanisms in organic photovoltaic devices upon exposure to a temperate and a subequatorial climate," Chem. Phys. Lett., vol. 640, pp. 201-214, Jan. 2015.

[11] P. Sonar, S. P. Singh, Y. Li, Z.-E. Ooi, T.-j. Ha, I. Wong, et al., "High mobility organic thin film transistor and efficient photovoltaic devices using versatile donoracceptor polymer semiconductor by molecular design," Energy Environ Sci, vol. 4, pp. 2288-2296, 2011.

[12] S. K. Hau, H.-L. Yip, N. S. Baek, J. Zou, K. O’Malley, and A. K.-Y. Jen, "Air-stable inverted flexible polymer solar cells using zinc oxide nanoparticles as an electron selective layer," Appl. Phys. Lett., vol. 92, p. 253301, 2008.

[13] S. B. Srivastava, P. Sonar, and S. P. Singh, "Charge transport studies in donoracceptor block copolymer PDPP-TNT and PC71BM based inverted organic photovoltaic devices processed in room conditions," $A I P A d v$., vol. 5, p. 077177, 2015. 
[14] F. A. Angel, Y. L. Lyubarskaya, A. A. Shestopalov, and C. W. Tang, "Degradation of self-assembled monolayers in organic photovoltaic devices," Org. Electron., vol. 15, pp. 3624-3631, 2014.

[15] B. Y. Finck and B. J. Schwartz, "Understanding the origin of the S-curve in conjugated polymer/fullerene photovoltaics from drift-diffusion simulations," Appl. Phys. Lett., vol. 103, p. 053306, 2013.

[16] B. J. Leever, C. A. Bailey, T. J. Marks, M. C. Hersam, and M. F. Durstock, "In Situ Characterization of Lifetime and Morphology in Operating Bulk Heterojunction Organic Photovoltaic Devices by Impedance Spectroscopy," Adv. Energy Mater., vol. 2, pp. 120-128, 2012.

[17] T. Kirchartz, W. Gong, S. A. Hawks, T. Agostinelli, R. C. I. MacKenzie, Y. Yang, et al., "Sensitivity of the Mott-Schottky Analysis in Organic Solar Cells," J. Phys. Chem. C, vol. 116, pp. 7672-7680, Apr. 2012.

[18] F. Deledalle, P. Shakya Tuladhar, J. Nelson, J. R. Durrant, and T. Kirchartz, "Understanding the Apparent Charge Density Dependence of Mobility and Lifetime in Organic Bulk Heterojunction Solar Cells," J. Phys. Chem. C, vol. 118, pp. 88378842, 2014.

[19] J. Bisquert, "Interpretation of electron diffusion coefficient in organic and inorganic semiconductors with broad distributions of states," PCCP, vol. 10, pp. 3175-3194, 2008.

[20] J. Bisquert and G. Garcia-Belmonte, "On Voltage, Photovoltage, and Photocurrent in Bulk Heterojunction Organic Solar Cells," J. Phys. Chem. Lett., vol. 2, pp. 19501964, Aug. 2011.

[21] B. Ray, A. G. Baradwaj, M. R. Khan, B. W. Boudouris, and M. A. Alam, "Collectionlimited theory interprets the extraordinary response of single semiconductor organic solar cells," PNAS, vol. 112 (36), pp. 11193-11198, Sep. 2015

[22] J. Bisquert, G. Garcia-Belmonte, P. Bueno, E. Longo, and L. O. S. Bulhões, "Impedance of constant phase element (CPE)-blocked diffusion in film electrodes," $J$. Electroanal. Chem., vol. 452, pp. 229-234, Jul. 1998. 
[23] L. Xu, Y.-J. Lee, and J. W. P. Hsu, "Charge collection in bulk heterojunction organic photovoltaic devices: An impedance spectroscopy study," Appl. Phys. Lett., vol. 105, p. 123904, 2014.

[24] A. Guerrero, S. Loser, G. Garcia-Belmonte, C. J. Bruns, J. Smith, H. Miyauchi, et al., "Solution-processed small molecule:fullerene bulk-heterojunction solar cells: impedance spectroscopy deduced bulk and interfacial limits to fill-factors," $P C C P$, vol. 15, pp. 16456-16462, 2013.

[25] S. Khelifi, K. Decock, J. Lauwaert, H. Vrielinck, D. Spoltore, F. Piersimoni, et al., "Investigation of defects by admittance spectroscopy measurements in poly (3hexylthiophene):(6,6)-phenyl C61-butyric acid methyl ester organic solar cells degraded under air exposure," J. Appl. Phys., vol. 110, p. 094509, 2011. 